\title{
OPEN Protection against SARS-CoV-2 by BCG vaccination is not supported by epidemiological analyses
}

\author{
Janine Hensel ${ }^{1,6}$, Kathleen M. McAndrews ${ }^{1,6}$, Daniel J. McGrail ${ }^{2,6}{ }^{2}$ Dara P. Dowlatshahi ${ }^{1}$, \\ Valerie S. LeBleu ${ }^{1,3}$ \& Raghu Kalluri $1,4,5 \square$
}

The Bacillus Calmette-Guerin (BCG) vaccine provides protection against tuberculosis (TB), and is thought to provide protection against non-TB infectious diseases. BCG vaccination has recently been proposed as a strategy to prevent infection with SARS-CoV-2 (CoV-2) to combat the COVID-19 outbreak, supported by its potential to boost innate immunity and initial epidemiological analyses which observed reduced severity of COVID-19 in countries with universal BCG vaccination policies. Seventeen clinical trials are currently registered to inform on the benefits of BCG vaccinations upon exposure to CoV-2. Numerous epidemiological analyses showed a correlation between incidence of COVID-19 and BCG vaccination policies. These studies were not systematically corrected for confounding variables. We observed that after correction for confounding variables, most notably testing rates, there was no association between BCG vaccination policy and COVD-19 spread rate or percent mortality. Moreover, we found variables describing co-morbidities, including cardiovascular death rate and smoking prevalence, were significantly associated COVID-19 spread rate and percent mortality, respectively. While reporting biases may confound our observations, our epidemiological findings do not provide evidence to correlate overall BCG vaccination policy with the spread of CoV-2 and its associated mortality.

Since the early 1920s, the use of the Bacillus Calmette-Guerin (BCG) vaccine has been implemented in several countries to prevent tuberculosis (TB), a disease caused by Mycobacterium tuberculosis. Infection with Mycobacterium tuberculosis primarily impacts lung function, and may lead to TB-related death. Immunization is done by exposing individuals to the M. tuberculosis-related, live, attenuated Mycobacterium bovis strain. The BCG vaccine thus offers a partial and possibly variable immunity against $\mathrm{TB}$, and its off-target effect has also been linked to protection against other infectious diseases ${ }^{1}$. The mechanism of protection against a broad range of pathogens has been linked to epigenetic reprogramming of monocytes and natural killer cells which then enables innate immune cells to fight a broad range of pathogens ${ }^{2}$.

In most countries with high TB incidence, a universal BCG vaccination policy has been implemented, where children receive the BCG vaccine in early infancy. In some of these countries, when TB incidence dropped, the universal policy was discontinued. In addition, a few countries have never implemented a universal BCG policy due to low TB incidence and the possibility to retain use of the tuberculin skin test in order to assess current TB infections. In countries with active BCG vaccination programs, the variability in protection against TB could be due to differences in the BCG strains used in vaccine synthesis, vaccine batch differences, time of vaccination, previous exposure to mycobacterium, and distinct host haplotypes ${ }^{3}$. The BCG vaccine may offer protection for as long as 30-40 years post vaccination (as previously reported in Norway ${ }^{4}$ ), and possibly as long as 50-60 years, as reported in American Indians and Alaska Natives that participated in a BCG vaccine trial ${ }^{5}$. However, these studies also show a reduction in long-lasting protection which is in line with studies showing that the protective effect of the BCG vaccine is decreased over time (reviewed in ${ }^{6}$ ).

\footnotetext{
${ }^{1}$ Department of Cancer Biology, Metastasis Research Center, University of Texas MD Anderson Cancer Center, Houston, TX, USA. ${ }^{2}$ Department of Systems Biology, University of Texas MD Anderson Cancer Center, Houston, TX, USA. ${ }^{3}$ Feinberg School of Medicine, Northwestern University, Chicago, IL, USA. ${ }^{4}$ Department of Bioengineering, Rice University, Houston, TX, USA. ${ }^{5}$ Department of Molecular and Cellular Biology, Baylor College of Medicine, Houston, TX, USA. ${ }^{6}$ These authors contributed equally: Janine Hensel, Kathleen M. McAndrews and Daniel J. McGrail. ${ }^{\bowtie}$ email: rkalluri@mdanderson.org
} 


\begin{tabular}{|c|c|c|c|c|c|}
\hline Clinical trial number & Title & Geographic location & Cohort Size & BCG strain & BCG vaccination policy \\
\hline NCT04347876 & $\begin{array}{l}\text { Outcome of COVID-19 Cases Based on } \\
\text { Tuberculin Test: Can Previous BCG Alter } \\
\text { the Prognosis? }\end{array}$ & Egypt & 100 & NA & Current \\
\hline NCT04350931 & $\begin{array}{l}\text { Application of BCG Vaccine for Immune- } \\
\text { prophylaxis Among Egyptian Health- } \\
\text { care Workers During the Pandemic of } \\
\text { COVID-19 }\end{array}$ & Egypt & 900 & unknown & Current \\
\hline NCT04461379 & $\begin{array}{l}\text { Prevention, Efficacy and Safety of BCG } \\
\text { Vaccine in COVID-19 Among Healthcare } \\
\text { Workers }\end{array}$ & Mexico & 908 & Tokio 172 strain & Current \\
\hline NCT04379336 & $\begin{array}{l}\text { BCG Vaccination for Healthcare Workers } \\
\text { in COVID-19 Pandemic }\end{array}$ & South Africa & 500 & Danish strain 1331, SSI, Denmark & Current \\
\hline NCT04362124 & $\begin{array}{l}\text { Performance Evaluation of BCG Vaccina- } \\
\text { tion in Healthcare Personnel to Reduce } \\
\text { the Severity of SARS-COV-2 Infection }\end{array}$ & Columbia & 1000 & BCG Liofilizada & Current \\
\hline NCT04475302 & $\begin{array}{l}\text { BCG Vaccine in Reducing Morbidity } \\
\text { and Mortality in Elderly Individuals in } \\
\text { COVID-19 Hotspots }\end{array}$ & India & 2175 & Serum Institute of India & Current \\
\hline NCT04369794 & $\begin{array}{l}\text { COVID-19: BCG As Therapeutic Vaccine, } \\
\text { Transmission Limitation, and Immuno- } \\
\text { globulin Enhancement (BATTLE) }\end{array}$ & Brazil & 1000 & Calmette Guerin bacillus & Current \\
\hline NCT04327206 & $\begin{array}{l}\text { BCG Vaccination to Protect Healthcare } \\
\text { Workers Against COVID-19 (BRACE) }\end{array}$ & Australia & 10,078 & Danish strain 1331, SSI, Denmark & Past \\
\hline NCT04542330 & $\begin{array}{l}\text { Using BCG to Protect Senior Citizens } \\
\text { During the COVID-19 Pandemic }\end{array}$ & Denmark & 1900 & Danish strain 1331, SSI, Denmark & Past \\
\hline NCT04373291 & $\begin{array}{l}\text { Using BCG Vaccine to Protect Health } \\
\text { Care Workers in the COVID-19 Pandemic }\end{array}$ & Denmark & 1500 & Danish strain 1331, SSI, Denmark & Past \\
\hline NCT04384549 & $\begin{array}{l}\text { Efficacy of BCG Vaccination in the Pre- } \\
\text { vention of COVID19 Via the Strengthen- } \\
\text { ing of Innate Immunity in Health Care } \\
\text { Workers (COVID-BCG) }\end{array}$ & France & 1120 & unknown & Past \\
\hline NCT04414267 & $\begin{array}{l}\text { Bacillus Calmette-guérin Vaccination to } \\
\text { Prevent COVID-19 (ACTIVATEII) }\end{array}$ & The Netherlands & 900 & $\begin{array}{l}\text { BCG vaccine Moscow strain } 361-1 ; \\
\text { Serum Institute of India Pvt. Ltd }\end{array}$ & Never \\
\hline NCT04417335 & \begin{tabular}{l|} 
Reducing COVID-19 Related Hospital \\
Admission in Elderly by BCG Vaccination
\end{tabular} & The Netherlands & 2014 & Danish strain 1331, SSI, Denmark & Never \\
\hline NCT04537663 & $\begin{array}{l}\text { Prevention Of Respiratory Tract Infection } \\
\text { And Covid-19 Through BCG Vaccination } \\
\text { In Vulnerable Older Adults (BCG- } \\
\text { PRIME) }\end{array}$ & The Netherlands & 5200 & Danish strain 1331, SSI, Denmark & Never \\
\hline NCT04328441 & $\begin{array}{l}\text { Reducing Health Care Workers Absentee- } \\
\text { ism in Covid-19 Pandemic Through BCG } \\
\text { Vaccine (BCG-CORONA) }\end{array}$ & The Netherlands & 1500 & unknown & Never \\
\hline NCT04534803 & $\begin{array}{l}\text { BCG Against Covid-19 for Prevention } \\
\text { and Amelioration of Severity Trial (BAC } \\
\text { to the PAST) }\end{array}$ & USA & 2100 & Tokyo-172 Strain & Never \\
\hline NCT04348370 & $\begin{array}{l}\text { BCG Vaccine for Health Care Workers as } \\
\text { Defense Against COVID } 19 \text { (BADAS) }\end{array}$ & USA & 1800 & BCG Tice strain & Never \\
\hline
\end{tabular}

Table 1. Registered clinical trials for COVID-19 related studies.

There is evidence from previous outbreaks that supports the hypothesis that BCG vaccination can protect against RNA and DNA viral infections (herpes and influenza) and other diseases such as asthma, as well as lowers yellow fever viremia ${ }^{2,7,8}$. Encouraging data also emerged from a recent double-blind, randomized clinical trial, showing that patients $\geq 65$ years vaccinated with BCG had a reduced incidence of infections, in particular respiratory tract infections ${ }^{9}$. This study is in line with other previous reports showing a reduction in respiratory tract infections in the BCG vaccinated cohort ${ }^{10,11}$.

Preliminary epidemiological analyses have shown BCG vaccination is associated with reduced COVID-19 cases and mortality ${ }^{12-17}$. These observations have given support to the possibility of the BCG vaccine providing protection against infection with the novel coronavirus, SARS CoV-2 (CoV-2). In consideration for the potential protection of BCG vaccination against other infectious diseases including CoV-2 infection, seventeen clinical trials assessing the efficacy of BCG vaccination at preventing COVID-19 have been registered (Table 1). However, many of the epidemiological observations do not account for potential confounding variables, including socioeconomic factors, co-morbidities, and CoV-2 testing rate. In this study, we systematically analyzed whether the BCG vaccination policy of countries correlates with COVID-19 spread and mortality after adjusting for confounding variables.

\section{Methods}

We collected information for BCG vaccination policies, BCG strains, TB incidence and BCG coverage across all countries from the BCG World Atlas (https://www.bcgatlas.org/). For analysis of countries with past BCG vaccination policy, countries with a year BCG was introduced and stopped were included. The BCG World Atlas 
a

Never universal BCG policy

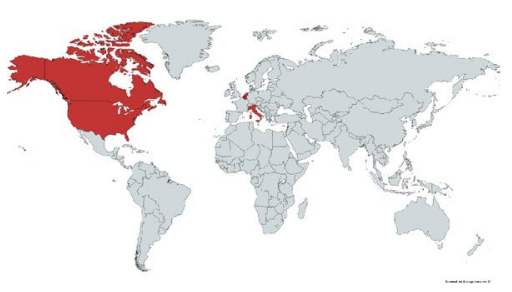

b

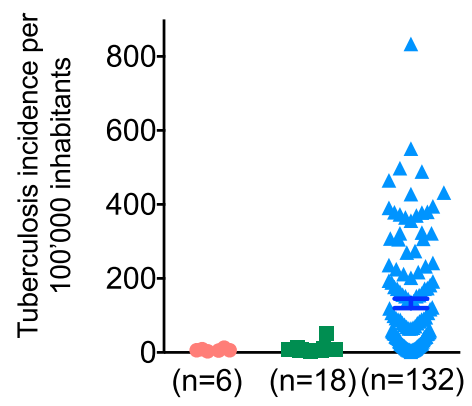

$\left.\left.\begin{array}{l}\text { - Never universal BCG policy } \\ \text { - Universal BCG policy in the past } \\ \text { - Current universal BCG policy }\end{array}\right] p=1.3 \times 10^{-3}\right] p=7.3 \times 10^{-2}$
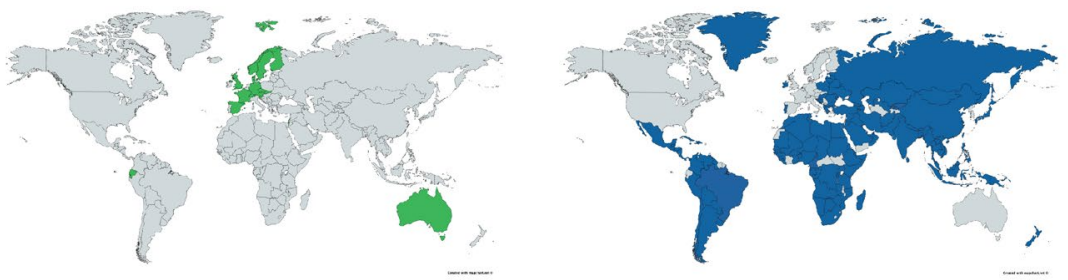

C

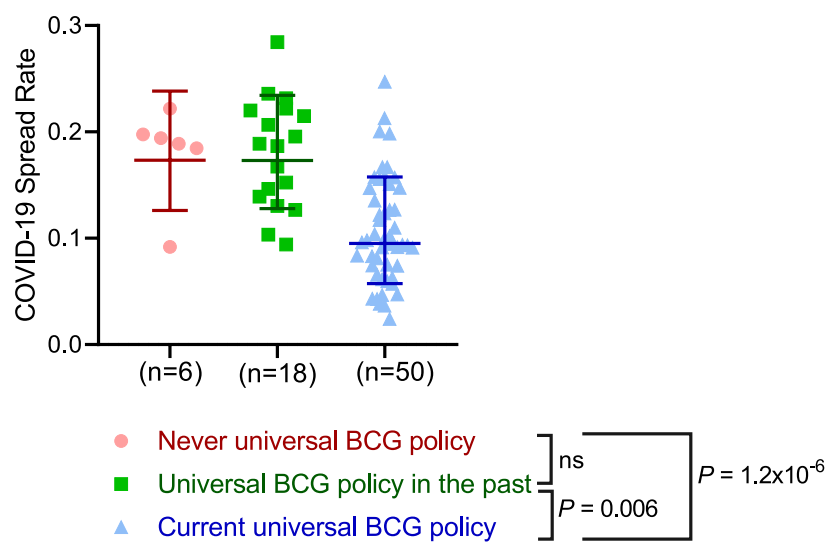

Figure 1. COVID-19 spread rate in countries with distinct national BCG vaccination policy. (a) World maps show countries that never had a national BCG vaccination policy (in red), countries that had a universal BCG vaccination policy in the past (in green) and countries that currently have a universal BCG vaccination policy (in blue). (b) TB incidence per 100,000 inhabitants shown as mean \pm SEM for all three groups of BCG policies (never (red), past (green) and current (blue) BCG vaccination policy). One-way ANOVA with Tukey posttest was performed. (c) CoV-2 spread rate is shown for all three groups of BCG policies (never (red), past (green) and current (blue) BCG vaccination policy). Data shown as median \pm interquartile range. Maps were generated using the following website: https://mapchart.net/world.html.

was last updated in 2017. Data of total COVID-19 cases, new COVID-19 cases, first reported COVID-19 cases, mortality, and testing rates for each country was obtained June 10th, 2020 from the COVID-19 Data Repository by the Center for Systems Science and Engineering (CSSE) at Johns Hopkins University (https://github.com/ CSSEGISandData/COVID-19). Total population, population density (population $/ \mathrm{km}^{2}$ ), fraction of population over 65, fraction of the population that lives in urban areas, per capita GDP, smoking rates, diabetes prevalence, and cardiovascular disease related mortality were obtained from Our World in Data (https://ourworldindata. org). World maps were generated using: https://mapchart.net/world.html.

Data were analyzed using Matlab R2016a. For COVID-19 spread rates, data were modeled to an exponential growth equation, Cases $\sim$ Cases $0 \cdot \exp \left(k_{\text {spread }} \cdot t\right)$, where Cases is cases per million inhabitants, Cases 0 is the number of cases per million inhabitants at the initial time point, $k_{\text {spread }}$ is the COVID-19 spread rate, and $t$ is the time point in days. The initial time point was considered as when countries exceeded 1 case per million inhabitants, and the spread rate was fit over the first 21 days after COVID-19 cases reached 1 per million inhabitants. Univariate and multivariate regression analysis was performed on COVID-19 spread rates and percent mortality, defined as deaths per total COVID-19, cases using the fitglme function. All confounding variables were z-normalized for equivalent scaling, and p-values corrected using Benjamini and Hochberg procedure for univariate comparisons. For primary analyses, BCG vaccination (BCG Vac.) was treated as continuous variable divided between 3 discrete levels, 0 for never universal BCG vaccination policy, 1 for universal BCG vaccination policy in the past, and 2 for current universal BCG vaccination policy. Additional analyses were performed using two discrete levels for BCG vaccination policy, dividing into never or past universal BCG vaccination policy compared to current BCG vaccination policy. For some comparisons, countries were limited to those with testing rates of 10 tests per thousand and greater, as specified in the results.

\section{Results}

As of June 13th, 2020, 7,713,571 CoV-2 infected individuals have been confirmed across the globe. Interestingly, 17 clinical trials are currently registered to evaluate BCG vaccination for COVID-19 on https://clinicaltrials. gov (Table 1). Seven trials are initiated in countries that currently have a universal BCG policy in place (Egypt, Mexico, South Africa, Columbia, India and Brazil; Table 1). Four trials are conducted in countries that had a 
a

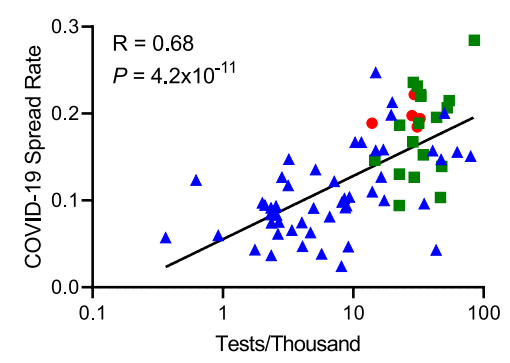

4. Current universal BCG policy

- Universal BCG policy in the past

C

- No universal BCG policy

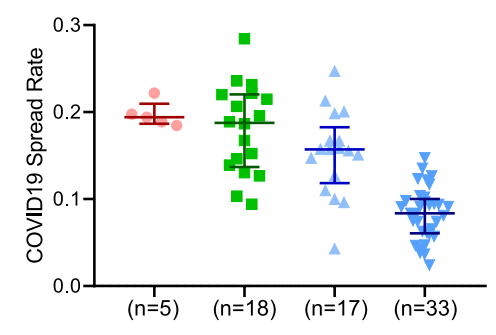

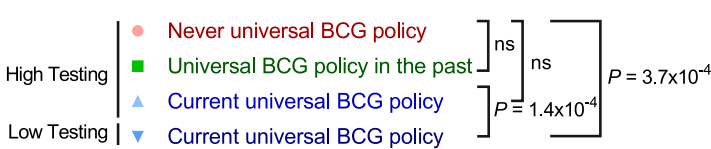

e

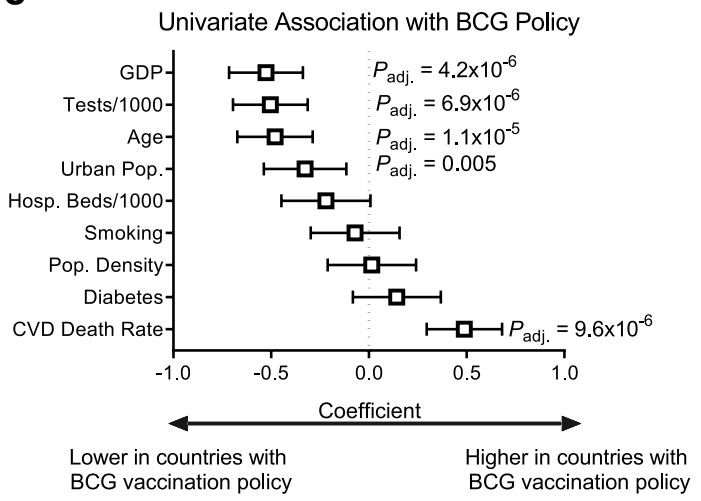

b

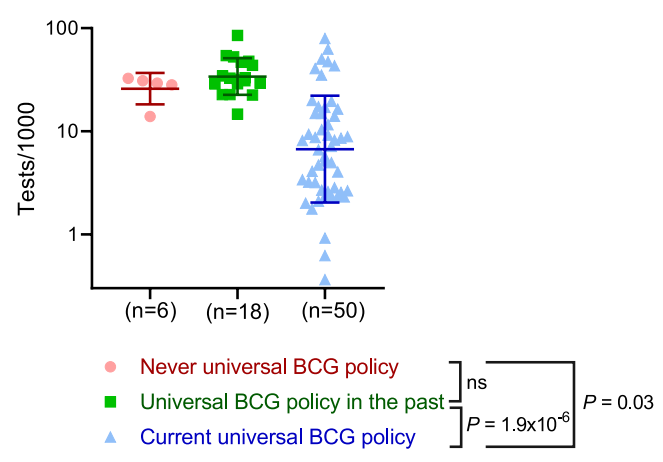

d

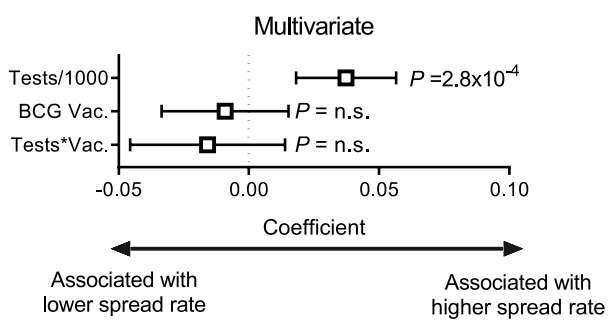

f

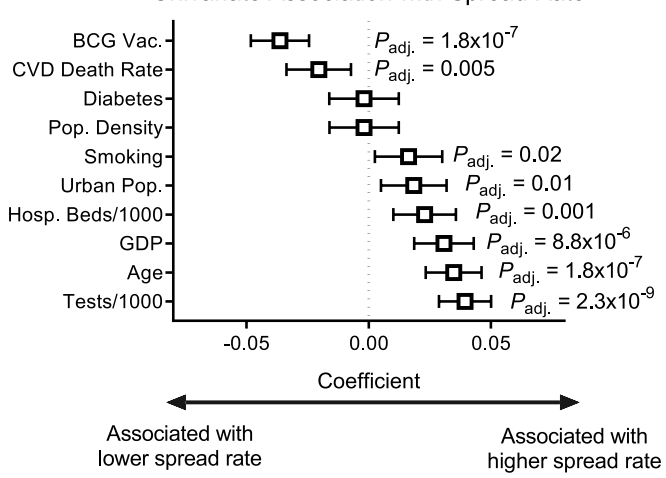

g

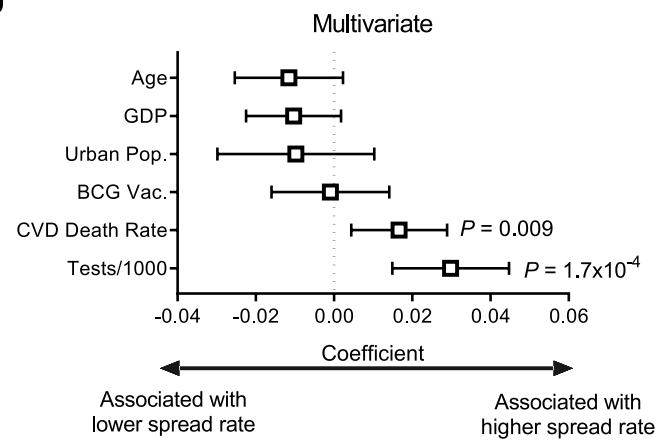


4 Figure 2. SARS-CoV-2 testing rates influence the observed benefit of BCG vaccination policy. (a) Correlation graph of total COVID-19 spread rate and tests per thousand inhabitants. Inset value is Spearman correlation coefficient $(\rho)$. (b) Tests/1000 is shown for all three groups of BCG policies (never (red), past (green) and current (blue) BCG vaccination policy) comparing high and low testing rate. Data shown as median with interquartile range. Kruskal-Wallis with Dunn's post-hoc test performed. (c) CoV-2 spread rate is shown for all three groups of BCG policies (never (red), past (green) and current (blue) BCG vaccination policy) comparing high and low testing rate. Data shown as median with interquartile range. Kruskal-Wallis with Dunn's post-hoc test performed. (d) Multivariate regression analysis of CoV-2 spread rate shows the coefficients and $p$ values for tests per 1000 inhabitants. (e) Univariate analysis of associations with BCG vaccination policy shows the coefficients and adjusted $p$ values for cardiovascular disease (CVD) death rate, diabetes population density (pop. density), smoking rate, urban population (urban pop.), hospital beds per 1000 inhabitants, gross domestic product (GDP), age and tests per 1000 inhabitants. (f) Univariate analysis of associations with CoV-2 spread rate shows the coefficients and adjusted $p$ values for cardiovascular disease (CVD) death rate, diabetes population density (pop. density), smoking rate, urban population (urban pop.), hospital beds per 1000 inhabitants, gross domestic product (GDP), age and tests per 1000 inhabitants. (g) Multivariate regression analysis of CoV-2 spread rate for variables significant in $2 \mathrm{e}$ and $2 \mathrm{f}$.

universal BCG policy in the past (Australia, Denmark, and France) and 6 trials are in the Netherlands and USA, two countries that never had a universal BCG policy (Table 1). The largest study, based on numbers of participants, is underway in Australia to study the effects of BCG vaccination on COVID-19 incidence, duration and severity of disease.

Intrigued by the extensive number of clinical trials initiated, we wanted to analyze currently available data to test the association between BCG vaccination policy and CoV-2 spread rate and its associated mortality. BCG vaccination policy information is available for 158 countries (Supplementary Table S1). Belgium, Netherlands, Canada, Italy, Lebanon, and United States of America never had a universal BCG policy, yet all these countries offer vaccination for high risk subgroups (healthcare professionals and children with parents from high risk countries) ('Never universal GCG policy', Fig. 1a). 18 countries had a universal BCG policy in the past, including many European countries, Australia, Ecuador, and Israel ('Universal BCG policy in the past', Fig. 1a). 134 countries currently have a universal BCG vaccination policy, including most countries in Central America, South America, Africa, and Asia ('Current universal BCG policy', Fig. 1a). First, we analyzed TB incidence in all countries with BCG policy information. Two countries with BCG policy information had no TB incidence numbers listed on the BCG World Atlas and therefore were not included in our analysis. Our analysis showed that countries with an active universal BCG policy have higher TB incidence as compared to countries that had a universal BCG in the past (Fig. 1b). We had complete data available for 74 countries to study the association of BCG vaccination policy and COVID-19 spread and mortality. The COVID-19 spread rate was significantly reduced in countries with current universal BCG policy when compared to countries with a never- or in the past-universal BCG policy (Fig. 1c).

The administered BCG vaccination strain did not account for a higher mortality per 100,000 inhabitants (Supplementary Figure S1a). Spain and Sweden had a high mortality per 100,000 inhabitants and both countries had a universal BCG policy in the past. Based on this observation we analyzed whether the year in which the BCG policy was discontinued correlates with mortality. We found that the mortality per 100,000 inhabitants was not correlated with the time the universal BCG policy was discontinued (Supplementary Figure S1b). Next, we analyzed mortality in countries with current universal BCG policy. There was no correlation observed in the mortality per 100,000 inhabitants and the time since implementation of the universal BCG policy (Supplementary Figure S1c). BCG coverage data was available for 51 countries which provides an estimate on access and adherence to BCG policy in each country (Supplementary Table S2). Except for Ukraine, which reported a coverage of $15-45 \%$, most countries report a coverage higher than $90 \%$.

While we observed decreased COVID-19 spread rate in countries with universal BCG vaccination policies, this analysis is fundamentally limited by the extent to which countries are capable of testing for CoV-2 to quantify spread of COVID-19. Comparison of COVID-19 spread rate and rates of testing yielded a strong positive correlation of 0.68 ( $p$ value $=4.2 \times 10^{-11}$ ) (Fig. $\left.2 \mathrm{a}\right)$. CoV-2 testing rates were significantly different between countries with distinct BCG vaccination policies (Fig. 2b). When only including "high CoV-2 testing" countries with 10 or more tests per thousand, resulting in equivalent distribution of $\mathrm{CoV}-2$ testing rates across countries with different BCG policies, COVID-19 spread rate is no longer associated with BCG policy (Fig. 2c). Consistent with this, multivariate analysis indicated testing rate, but not BCG vaccination policy, was associated with COVID-19 spread rate (Fig. 2d). To assess if additional factors could be confounding the analysis, we analyzed the association of population density (population $/ \mathrm{km}^{2}$ ), fraction of population over 65 , fraction of the population that lives in urban areas (urban pop.), per capita GDP, smoking rates, diabetes prevalence, and cardiovascular disease (CVD) related mortality with both BCG vaccination policy (Fig. 2e) and COVID-19 spread rate (Fig. 2f). Performing multivariate analysis of variables significantly associated with both BCG vaccination policy and COVID-19 spread rate indicated that testing rate and cardiovascular death rate were independently associated with increased COVID-19 spread rates (Fig. 2g). Percent COVID-19 mortality was significantly lower between countries that have a current universal BCG policy compared to countries that never had a universal BCG policy ( $p$ value $=0.01$ ) (Fig. 3a). However, univariate analysis showed that age confounds the analysis (Fig. 3b). When adjusted for age the percent CoV-2 mortality is not different in countries with different BCG policies, though age remained statistically associated with percent mortality $(P=0.001)$ (Fig. 3c). Repeating these analyses using BCG vaccination policy as a binary variable, that is current universal policy $v s$ no current universal policy, produced 
a

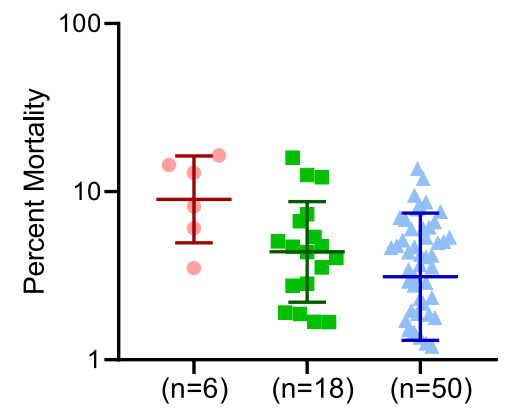

$\left.\left.\begin{array}{l}\text { Never universal BCG policy } \\ \text { - Universal BCG policy in the past } \\ \text { Current universal BCG policy }\end{array}\right] \mathrm{ns}\right] P=0.01$

b

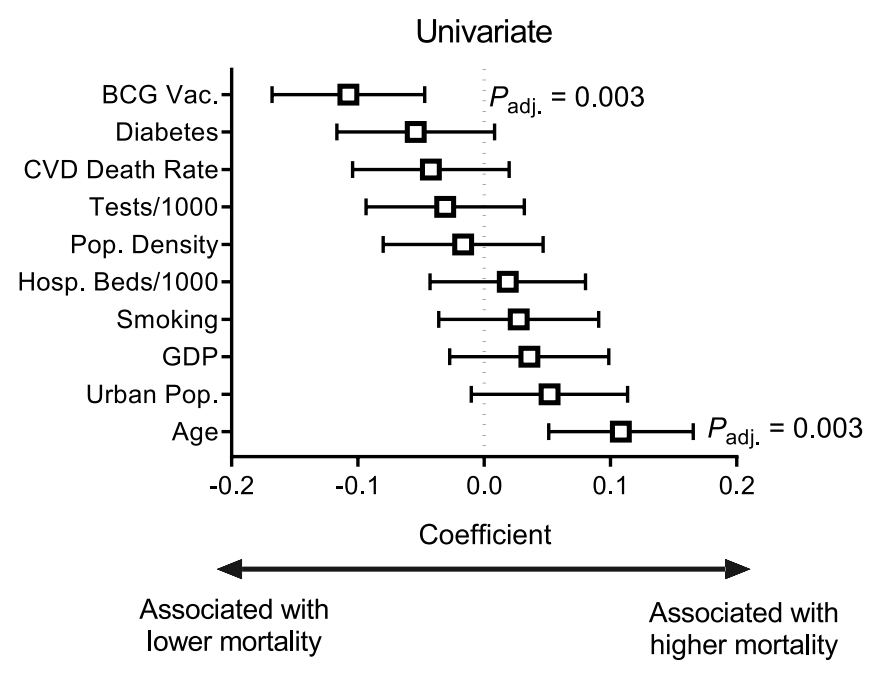

C

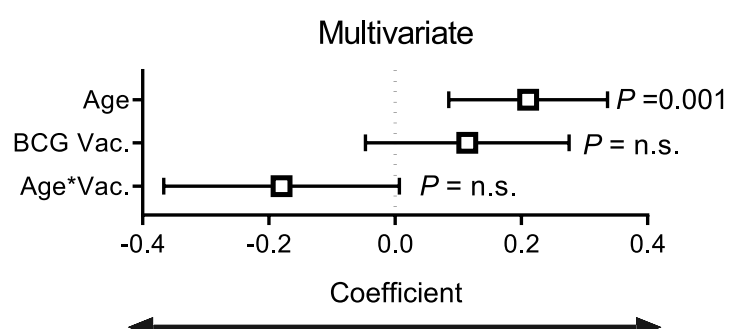

Associated with lower mortality

Associated with higher mortality

Figure 3. Distribution of COVID-19 mortality and BCG vaccination policy. (a) CoV-2 mortality is shown for all three groups of BCG policies (never (red), past (green) and current (blue) BCG vaccination policy). Data shown as median \pm interquartile range. (b) Univariate analysis of CoV-2 mortality shows the coefficients and adjusted $p$ values for BCG vaccination policy (BCG Vac.), diabetes, cardiovascular disease (CVD) death rate, tests per 1000 inhabitants, population density (pop. density), hospital beds per 1000 inhabitants, smoking rate, gross domestic product (GDP), urban population (urban pop.) and age. (c) Multivariate regression analysis of fraction of population over 65 and BCG vaccination policy with COVID19 percent mortality. 
equivalent results with the exception that there was no longer a significant association between BCG vaccination policy and COVID-19 mortality (Supplementary Figure S2).

To further confirm our observations, we next repeated our analyses only in European countries, aiming to increase the homogeneity in population genetics and demographic characteristics to improve the quality of our comparisons. Upon initial analysis, we still observed distinct demographic differences between countries with and without current BCG vaccination policies (Supplementary Figure S3a). To address this, we performed K-means clustering based on demographic descriptors (excluding BCG vaccination policy), dividing Europe into two clusters (Supplementary Figure S3b). All countries in Cluster 2 had a BCG vaccination policy, but Cluster 1 had 3 countries without BCG vaccination policies (Belgium, Italy, and Netherlands). Analysis of only Cluster 1 found no demographic features (population density, fraction of population over 65, fraction of the population that lives in urban areas, hospital beds per capita, per capita GDP, smoking rates, diabetes prevalence, or cardiovascular disease related mortality) to be associated with BCG vaccination policy (Supplementary Figure S3c). In countries from Cluster 1, BCG vaccination policy was not associated with changes in COVID-19 spread rate (unadjusted $P=0.86$ ) (Supplementary Figure S3d). Although no demographic features achieved $P<0.05$ following correction for multiple comparisons, tests/1000 inhabitants and smoking had unadjusted $P$ values $<0.05$. Performing multivariate regression analysis using these two parameters indicated that both are likely independently associated with an increase in observed COVID-19 spread rate (Supplementary Figure S3e). Analyzing the univariate association of percent mortality from COVID-19 within countries from Cluster 1 revealed both GDP and Tests/1000 were significantly $\left(P_{\text {adj. }}<0.05\right)$ associated with lower percent mortality, whereas age was only significant prior to correction for multiple comparisons (Supplementary Figure S3f). Multivariate regression analysis using three parameters indicated that smoking prevalence and tests/1000 inhabitants are likely independently associated with increased and decreased COVID-19 mortality, respectively (Supplementary Figure S3g).

\section{Discussion}

Our analysis of COVID-19 spread rate in 74 countries that were stratified based on the prevalence of BCG vaccination (no policy, prior universal vaccination policy, or current universal vaccination policy) shows that, a priori, there is a correlation between current universal BCG vaccination policy and a lower COVID-19 spread rate and mortality. Interestingly, we note that testing rates (tests per thousand inhabitants) was positively correlated with incidence of CoV-2 infection. Taking in consideration this important bias, we stratified the data to compare the CoV-2 spread rate amongst countries with distinct universal BCG policy based on high CoV-2 testing rates, as defined by 10 or more tests per thousand inhabitants. The results show a lack of significant difference in COVID-19 spread rate in countries with current universal BCG vaccination policy and countries with never or in the past universal BCG vaccination policy.

Other variables tested in our analyses included GDP, median age, percent urban population, hospital beds per 1,000 inhabitants, smoking, population density, diabetes and CVD related deaths. GDP, median age, percent urban population and CVD related deaths were identified as additional confounders. We did not observe higher COVID-19 mortality when stratifying countries based on vaccine strain, however, that could at least partially be due to limited sample size ( $n=44$ countries).

Our study is limited by several critical considerations, including the variability and inaccuracies in reporting positive cases and reporting specific mortality associated with CoV-2 infection and lack of reporting on asymptomatic or minimally symptomatic cases. There could be also differences at the subnational level, for example as seen in India where most reported CoV-2 cases are from 5 states, which limits conclusions for the entire country. It is possible that there are also some countries that have subnational differences, for example in accessing healthcare, which could influence $\mathrm{CoV}-2$ infection rates and are not accounted for in our analysis.

Another limitation could be the adherence and access to vaccination as per policy. BCG coverage data suggested a high coverage (>90\%) in most countries, therefore, countries are likely comparable in respect to BCG coverage. In our study, we address the impact of social and economic barriers in accessing care by including GDP and hospital beds per capita. Other studies used similar metrics, including the human development index (HDI), describing access to health and education ${ }^{18}$ and income ${ }^{17}$. Both studies show a correlation of universal BCG vaccination policies and the number of COVID-19 cases when controlling for HDI or income, however, neither of these studies took $\mathrm{CoV}-2$ testing rates into consideration.

In our study, we try to take into account variables describing co-morbidities such as smoking rates, diabetes prevalence and cardiovascular disease related mortality. There are many more co-morbidities listed by the Center of Disease Control and Prevention, including cancer and obesity which could be additional confounding variables but haven't been analyzed in our study.

Differences in the virus strains could also confound our analyses, which is supported by a recent study showing that individuals infected with the spike protein variant $\mathrm{G} 614$ have higher viral loads ${ }^{19}$. Suggested polymorphisms in ACE2 itself could further confound our analysis ${ }^{20}$. Interestingly, BCG vaccination status did not correlate with COVID-19 mortality even within the same geographical location as shown in two recent studies ${ }^{21,22}$. It is also possible that countries with higher temperatures have lower transmission rates as suggested in a recently published study, however, this is also an epidemiological study that is confounded by multiple variables ${ }^{23}$. Finally, time since first exposure rates, as well as population adherence to social distancing measures, may also influence the evolving COVID-19 data collection. To date, social distancing remains critical in slowing down the number of new cases over time ${ }^{24}$.

In our study, we provide evidence that CoV-2 testing rates is a major confounder in the association between BCG vaccination policy and COVID-19 spread rate. Our study cautions against epidemiological studies showing correlation between BCG vaccination policy and COVID-19 spread rate. Currently conducted clinical trials 
are necessary to provide scientific data to evaluate the hypothesis that the BCG vaccine is effective to lower COVID-19 incidence and severity.

Received: 14 July 2020; Accepted: 26 September 2020

Published online: 27 October 2020

\section{References}

1. Tanner, R., Villarreal-Ramos, B., Vordermeier, H. M. \& McShane, H. The humoral immune response to BCG vaccination. Front. Immunol. 10, 1317. https://doi.org/10.3389/fimmu.2019.01317 (2019).

2. Arts, R. J. W. et al. BCG vaccination protects against experimental viral infection in humans through the induction of cytokines associated with trained immunity. Cell Host Microbe 23, 89 e105-100 e105. https://doi.org/10.1016/j.chom.2017.12.010 (2018).

3. Dockrell, H. M. \& Smith, S. G. What have we learnt about BCG vaccination in the last 20 years?. Front. Immunol. 20, 20 (2017).

4. Nguipdop-Djomo, P. H. E., Rodrigues, L. C., Abubakar, I. \& Mangtani, P. Duration of BCG protection against tuberculosis and change in effectiveness with time since vaccination in Norway: A retrospective population-based cohort study. Lancet Infect. Dis. 20, 20 (2016).

5. Aronson, N. E. et al. Long-term efficacy of BCG vaccine in American Indians and Alaska Natives: A 60-year follow-up study. JAMA 291, 2086-2091. https://doi.org/10.1001/jama.291.17.2086 (2004).

6. Menzies, D. Interpretation of repeated tuberculin tests. Boosting, conversion, and reversion. Am. J. Respir. Crit. Care Med. 159, 15-21. https://doi.org/10.1164/ajrccm.159.1.9801120 (1999).

7. Moorlag, S., Arts, R. J. W., van Crevel, R. \& Netea, M. G. Non-specific effects of BCG vaccine on viral infections. Clin. Microbiol. Infect. 25, 1473-1478. https://doi.org/10.1016/j.cmi.2019.04.020 (2019).

8. Arnoldussen, D. L., Linehan, M. \& Sheikh, A. BCG vaccination and allergy: A systematic review and meta-analysis. J. Allergy Clin. Immunol. 127, 246-253 e241-221. https://doi.org/10.1016/j.jaci.2010.07.039 (2011).

9. Giamarellos-Bourboulis, E. et al. ACTIVATE: Randomized clinical trial of BCG vaccination against infection in the elderly. Cell https://doi.org/10.1016/j.cell.2020.08.051 (2020).

10. Nakayama, K., Monma, M., Fukushima, T., Ohrui, T. \& Sasaki, H. Tuberculin responses and risk of pneumonia in immobile elderly patients. Thorax 55, 867-869. https://doi.org/10.1136/thorax.55.10.867 (2000).

11. Ohrui, T., Nakayama, K., Fukushima, T., Chiba, H. \& Sasaki, H. Prevention of elderly pneumonia by pneumococcal, influenza and BCG vaccinations. Nihon Ronen Igakkai Zasshi 42, 34-36. https://doi.org/10.3143/geriatrics.42.34 (2005).

12. Levine, D. I. A shred of evidence that BCG vaccine may protect against COVID-19: Comparing cohorts in Spain and Italy. medRxiv. https://doi.org/10.1101/2020.06.05.20123539(2020).

13. Curtis, N., Sparrow, A., Ghebreyesus, T. A. \& Netea, M. G. Considering BCG vaccination to reduce the impact of COVID-19. Lancet 395, 1545-1546. https://doi.org/10.1016/S0140-6736(20)31025-4 (2020).

14. Shet, A., Ray, D., Malavige, N., Santosham, M. \& Bar-Zeev, N. Differential COVID-19-attributable mortality and BCG vaccine use in countries. medRxiv. https://doi.org/10.1101/2020.04.01.20049478 (2020).

15. Dayal, D. \& Gupta, S. Connecting BCG Vaccination and COVID-19: Additional Data. medRxiv. https://doi. org/10.1101/2020.04.07.20053272 (2020).

16. Sala, G. \& Miyakawa, T. Association of BCG vaccination policy with prevalence and mortality of COVID-19. medRxiv. https:// doi.org/10.1101/2020.03.30.20048165 (2020).

17. Miller, A. et al. Correlation between universal BCG vaccination policy and reduced morbidity and mortality for COVID-19: An epidemiological study. medRxiv. https://doi.org/10.1101/2020.03.24.20042937 (2020).

18. Escobar, L. E., Molina-Cruz, A. \& Barillas-Mury, C. BCG vaccine protection from severe coronavirus disease 2019 (COVID-19). Proc. Natl. Acad. Sci. USA 117, 17720-17726. https://doi.org/10.1073/pnas.2008410117 (2020).

19. Korber, B. et al. Tracking Changes in SARS-CoV-2 spike: Evidence that D614G increases infectivity of the COVID-19 virus. Cell 182, 812-827 e819. https://doi.org/10.1016/j.cell.2020.06.043 (2020).

20. Devaux, C. A., Rolain, J. M. \& Raoult, D. ACE2 receptor polymorphism: Susceptibility to SARS-CoV-2, hypertension, multi-organ failure, and COVID-19 disease outcome. J. Microbiol. Immunol. Infect. 53, 425-435. https://doi.org/10.1016/j.jmii.2020.04.015 (2020).

21. O’Neill, L. A. J. \& Netea, M. G. BCG-induced trained immunity: Can it offer protection against COVID-19?. Nat. Rev. Immunol. 20, 335-337. https://doi.org/10.1038/s41577-020-0337-y (2020).

22. Bluhm, R. \& Pinkovskiy, M. The BCG Vaccine Does Not Protect Against COVID-19: Applying an Economist's Toolkit to a Medical Question. https://voxeu.org/article/bcg-vaccine-does-not-protect-against-covid-19 (2020).

23. Sehxra, S. T., Salciccioli, J. D., Wiebe, D. J., Fundin, S. \& Baker, J. F. Maximum daily temperature, precipitation, ultra-violet light and rates of transmission of SARS-Cov-2 in the United States. Clin. Infect. Dis. https://doi.org/10.1093/cid/ciaa681 (2020).

24. Maier, B. F. \& Brockmann, D. Effective containment explains subexponential growth in recent confirmed COVID-19 cases in China. Science 368, 742-746. https://doi.org/10.1126/science.abb4557 (2020).

\section{Author contributions}

J.H., K.M.M, D.J.M. performed analyes. J.H., K.M.M, D.J.M., D.P.D., V.S.L. and R.K. wrote the manuscript.

\section{Competing interests}

The authors declare no competing interests.

\section{Additional information}

Supplementary information is available for this paper at https://doi.org/10.1038/s41598-020-75491-x.

Correspondence and requests for materials should be addressed to R.K.

Reprints and permissions information is available at www.nature.com/reprints.

Publisher's note Springer Nature remains neutral with regard to jurisdictional claims in published maps and institutional affiliations. 
(c) (i) Open Access This article is licensed under a Creative Commons Attribution 4.0 International cc) License, which permits use, sharing, adaptation, distribution and reproduction in any medium or format, as long as you give appropriate credit to the original author(s) and the source, provide a link to the Creative Commons licence, and indicate if changes were made. The images or other third party material in this article are included in the article's Creative Commons licence, unless indicated otherwise in a credit line to the material. If material is not included in the article's Creative Commons licence and your intended use is not permitted by statutory regulation or exceeds the permitted use, you will need to obtain permission directly from the copyright holder. To view a copy of this licence, visit http://creativecommons.org/licenses/by/4.0/.

(C) The Author(s) 2020 